\title{
Estudio preliminar de la ictiofauna y los hábitats acuáticos del Río Bajo Paraguá, Santa Cruz, Bolivia
}

\author{
Vladimir Fuentes Rojas ${ }^{1,3}$; Damián Ignacio Rumiz ${ }^{1,2}$ \\ ${ }^{1}$ Museo de Historia Natural Noel Kempff Mercado, Universidad Autónoma Gabriel René Moreno - UAGRM, \\ Casilla 2489, Santa Cruz, Bolivia \\ ${ }^{2}$ Wildlife Conservation Society, Av. Las Américas c/ Bumberque 349, Casilla 6272, Santa Cruz, Bolivia \\ ${ }^{3}$ Autor para correspondencia: Vladimir Fuentes, e-mail: vladimirfuentes@gmail.com
}

Fuentes, V.; Rumiz, D. I. Preliminary study of fish fauna and aquatic habitats in the Lower Paraguá River, Santa Cruz, Bolivia. Biota Neotrop., vol. 8, no. 1, Jan./Mar. 2008. Available from: <http://www.biotaneotropica. org.br/v8n1/en/abstract?article+bn01808012008>.

\begin{abstract}
With the aim of improving our knowledge about the fish fauna of the Lower Paragua River (Iténez or Guaporé basin), fish collections and physic-chemical analyses of water were conducted in three localities in northern Santa Cruz, on the western border of the Noel Kempff Mercado National Park. Sampling periods lasted 5 days on each of three sites (Piso Firme, Florida y El Refugio) and two seasons (high waters: April-May, low waters: September). By using various fishing methods, 4098 fish belonging to 124 species were collected, 35 of them were new geographic records for the National Park. From all the species, $56 \%$ belonged to the Characiformes, $22 \%$ to Siluriformes (34 spp.), 15\% Perciformes (18 spp. of cichlids), 5\% Gymnotiformes and $2 \%$ Synbranchiformes, Rajiformes and Beloniformes. Species richness was low in the upper course of the river (Piso Firme: 90 species, Florida: 71 species, El Refugio: 23 species) as well as oxygen levels which reached a critical minimum at El Refugio during low waters and increased cover of floating vegetation. The proportion of new geographic records and the uncertainties about fish distribution and abundance indicate the need to conduct monitoring actions associated to the management plans in the area.
\end{abstract}

Keywords: fish fauna, inventories, Itenez or Guaporé River, Noel Kempff Mercado National Park.

Fuentes, V.; Rumiz, D. I. Estudio preliminar de la ictiofauna y los hábitats acuáticos del Río Bajo Paraguá, Santa Cruz, Bolivia. Biota Neotrop., vol. 8, no. 1, jan./mar. 2008. Disponible en: <http://www.biotaneotropica. org.br/v8n1/es/abstract?article+bn01808012008>.

Resumen: A fin de conocer la riqueza íctica del Río Bajo Paraguá (cuenca del Iténez o Guaporé) se realizaron colectas de peces y análisis físico-químicos del agua en tres localidades del norte de Santa Cruz, sobre el límite oeste del Parque Noel Kempff Mercado. Los muestreos duraron 5 días en cada sitio (Piso Firme, Florida y El Refugio) y en dos épocas del año (aguas altas: Abril-Mayo; aguas bajas: Septiembre). Utilizando diferentes artes de pesca se capturaron 4098 peces pertenecientes a 124 especies, de las cuales 35 fueron nuevos registros para el Parque Noel Kempff Mercado. De las 124 especies, el 56\% correspondió al orden Characiformes, 22\% a Siluriformes (con $34 \mathrm{spp}$ ), 15\% a Perciformes (18 cíclidos), 5\% a Gymnotiformes y 2\% a Synbranchiformes, Rajiformes y Beloniformes. La riqueza de especies fue menor en el curso superior del río (Piso Firme: 90 especies, Florida: 71 especies y El Refugio: 23 especies), al igual que la concentración de oxígeno que en El Refugio alcanzó niveles críticos en la época de escaso caudal por la mayor cobertura de plantas flotantes. La proporción de nuevos registros geográficos y los interrogantes sobre distribución y abundancia de los peces del Rio Paraguá indican la necesidad de implementar monitoreos sistemáticos asociados a los planes de manejo de la zona.

Palabras clave: ictiofauna, inventarios, río Iténez o Guaporé, Parque Nacional Noel Kempff Mercado. 


\section{Introducción}

La mayor diversidad de peces de agua dulce del mundo se encuentra en Sudamérica, con un estimado de 8000 especies (Malabarba et al. 1998). De ellas, 635 han sido registradas para Bolivia, principalmente en las cuencas del Amazonas y Del Plata (Sarmiento \& Barrera 2003). En la subcuenca del río Iténez o Guaporé, cerca del límite entre Bolivia y Brasil, los inventarios de fauna para el Parque Nacional Noel Kempff Mercado reportaron 246 especies de peces (Sarmiento 1998, Schaefer 2000), incluyendo un muestreo sobre el Río Paraguá que registró 39 especies. El Río Paraguá drena la penillanura laterítica del escudo precámbrico de Santa Cruz y ha sido clasificado como un río de aguas negras aunque no es un típico ejemplo de esta clase (Killeen 1998, Navarro \& Maldonado 2002). En su curso inferior o 'Bajo Paraguá' forma llanuras aluviales con mayor diversidad de ambientes acuáticos y es la base de la subsistencia para las comunidades asentadas frente al Parque Noel Kempff. Con el desarrollo del ecoturismo, la pesca y los planes de manejo de recursos en la zona (Van Damme 2001), se hace necesario mejorar el conocimiento de la ictiofauna y ecología de estos ambientes. El presente trabajo reporta resultados de inventarios de peces y caracterizaciones ambientales realizadas en tres sitios del río Bajo Paraguá e intenta identificar factores que influyen en la riqueza y distribución de las especies.

\section{Materiales y Métodos}

La cuenca del Río Paraguá se extiende por unos 30.000 km² sobre el escudo precámbrico de los departamentos de Santa Cruz y Beni, abarcando mayormente elevaciones de $500 \mathrm{~m}$ en la penillanura laterítica y el borde de la meseta de Huanchaca, hasta $150 \mathrm{~m}$ en la llanura aluvial del Bajo Paraguá. El clima de la cuenca presenta un rango de temperatura media anual de 22 a $27^{\circ} \mathrm{C}$, una precipitación media de 1.200 a $1.900 \mathrm{~mm}$, y entre 3 y 6 meses secos por año. El estudio se enfocó en tres sitios del curso del Bajo Paraguá sobre el límite occidental del Parque Noel Kempff Mercado, uno cerca de la confluencia con el Río Tarvo (sitio de El Refugio), otro río abajo en Florida, y el tercero en Piso Firme, cerca de la desembocadura en el Río Iténez (Figura 1). La vegetación predominante alrededor del río incluye bosques ribereños de tierra firme, sabanas húmedas e inundables y bosques inundables, todos florísticamente diversos y la mayoría estacionalmente influenciados por las crecientes (Killeen 1998).

Las colectas de peces se realizaron a lo largo de aproximadamente 800 metros del río y durante cinco días en cada sitio, en la época de aguas altas (Abril-Mayo) y la de aguas bajas (Septiembre) del año 1999. Como artes de pesca se utilizaron redes agalleras en los sitios más profundos y redes de arrastre en los sitios menores a $1 \mathrm{~m}$ de profundidad (Figura 2). Además se utilizaron redes de mano, cedazos y anzuelos, principalmente para la pesca nocturna. Las diferentes artes de pesca fueron aplicadas con similar esfuerzo en cada muestreo para luego comparar la riqueza y abundancia de las capturas. En los sitios de colecta se describió la vegetación, se tomaron datos físicos y químicos; como transparencia del agua con un disco de Secchi, temperatura con un termómetro de mercurio, $\mathrm{pH}$ y oxígeno disuelto con la ayuda de un equipo Hach. La temperatura y $\mathrm{pH}$ fueron registradas cada 6 horas durante un día en cada sitio y época, y se calculó su media.

Los peces capturados fueron contados, identificados preliminarmente, registrados por sitio, y seleccionados para preservar en una muestra en formol. Para su identificación se contó una lupa estereoscópica y diferentes claves taxonómicas específicas para cada grupo de peces (Goulding 1980, Gery 1977, Kullander 1986, Mago-Lecia 1994, Vari 1984, 1991, 1992, Eigenmann 1890, Machado-Allison 1995, Malabarba 1998, Lauzanne 1985, Nelson 1999). Los especimenes fueron catalogados e incorporados a la colección húmeda del Museo

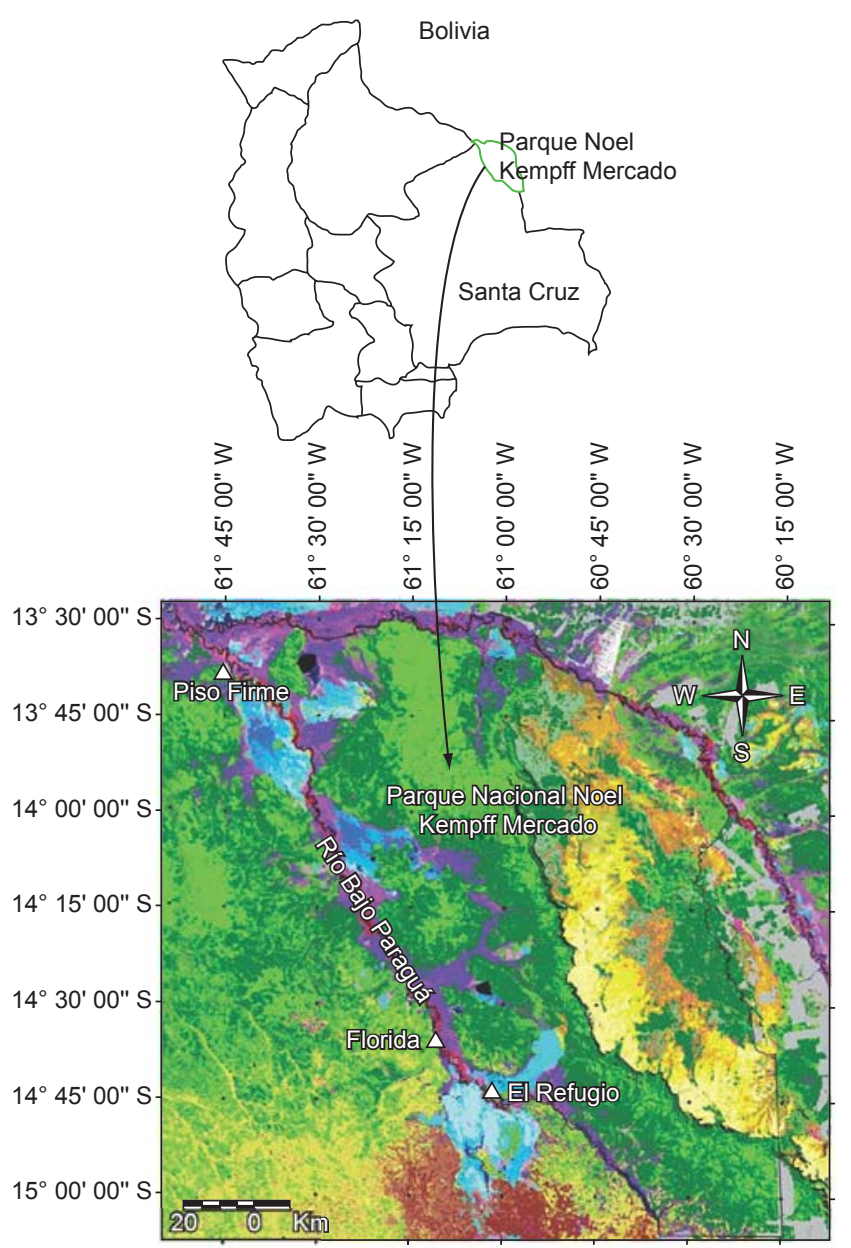

Figura 1. Mapa de ubicación.

Figure 1. Map of location.

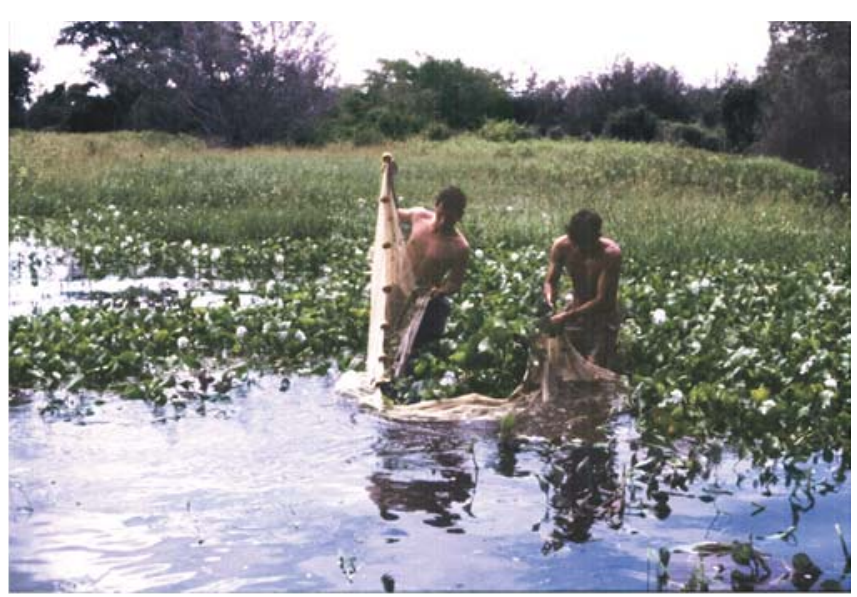

Figura 2. Colecta de peces en áreas de inundación de Florida.

Figure 2. Fish collection in flooded areas of Florida.

Noel Kempff Mercado. Con el número de individuos capturados y las especies identificadas se examinó la variación de la abundancia y riqueza de peces entre épocas y sitios así como su similaridad con el índice de Sorensen (Magurran 1989). Finalmente, se confeccionó una lista de especies registradas, adicionando las previamente mencionadas por Sarmiento (1998) en la localidad de El Refugio. 


\section{Resultados}

\section{Caracterización ambiental del río Bajo Paraguá y los sitios muestreados}

El trayecto del Río Bajo Paraguá en El Refugio (confluencia con el Tarvo) está rodeado de sabanas o pampas y escaso bosque ribereño casi hasta Florida (Figura 3), desde donde luego predomina el bosque inundable de igapó (Figura 4) y otros bosques hasta llegar

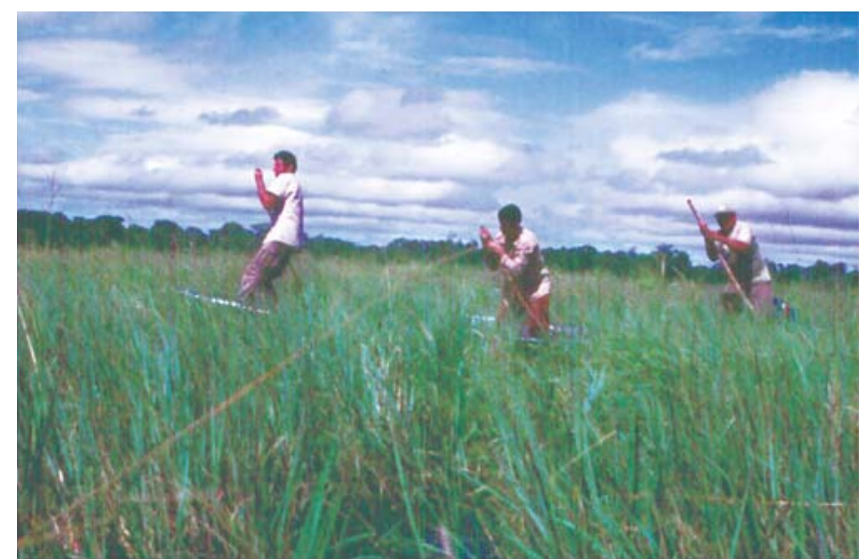

Figura 3. Cauce principal del Río Paraguá en la zona de El Refugio (pampas).

Figure 3. Main channel of the Paraguá River in El Refugio zone (savannas).

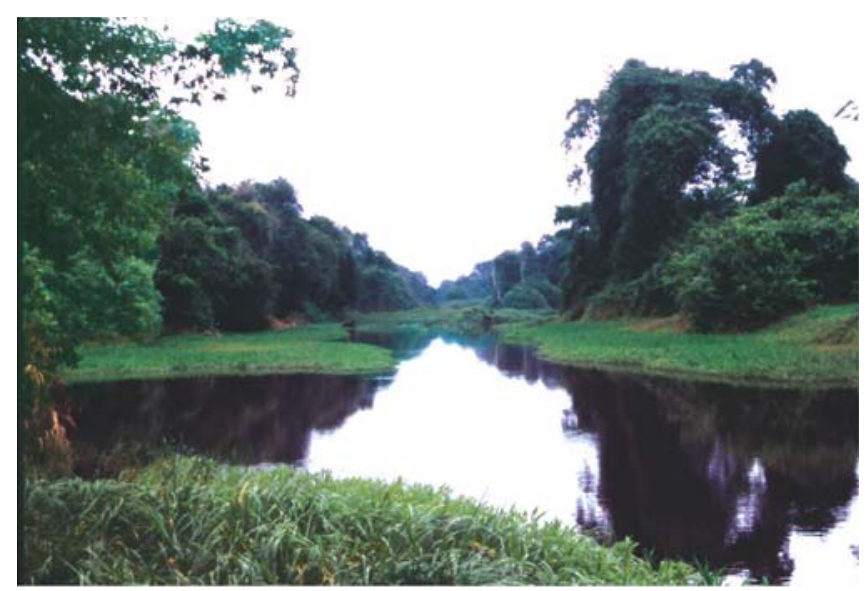

Figura 4. Cauce principal del Río Paraguá, zona Florida (Bosque de Igapo).

Figure 4. Main channel of the Paraguá River, Florida zone (Forest of Igapo). a Piso Firme. Las aguas del Río Bajo Paraguá son de color marrón de una alta transparencia (110 a $170 \mathrm{~cm})$, con escasas partículas en suspensión, corriente lenta (flujo laminar), y una profundidad máxima registrada que varió entre $2 \mathrm{~m}$ en la época de aguas bajas en El Refugio y $7 \mathrm{~m}$ en época de aguas altas en Florida. El mayor ancho del cauce durante las aguas bajas se registró en Piso Firme con $100 \mathrm{~m}$ y el menor en El Refugio con $30 \mathrm{~m}$; mientras que durante las aguas altas la zona de inundación de bosque y sabanas llegó hasta 1 km. Los niveles medios de oxígeno disuelto disminuyeron consistentemente río arriba, presentando poca variación en el canal principal durante la época de aguas altas (Piso Firme 6.5, Florida 5.8, y El Refugio $5.6 \mathrm{mg} . \mathrm{l}^{-1}$ ) y un marcado gradiente durante aguas bajas $(5.57,3.83 \mathrm{y}$ $0.9 \mathrm{mg} .1^{-1}$, respectivamente). En El Refugio, el canal principal del río alcanzó una escasez crítica de oxígeno durante aguas bajas, aunque simultáneamente una de sus 'bahías' o meandros dió una medición de $7 \mathrm{mg} . \mathrm{l}^{-1}$ (Tabla 1 ).

La vegetación flotante de 'tarope' (Eichhornia azurea) y 'yomomo' (Paspalum repens) predominó en las orillas y a menudo cubría totalmente el curso del río durante la época de aguas bajas. Durante las aguas altas, estas 'colchas' se redujeron al dispersarse en una mayor superficie de agua y fueron eliminadas del cauce principal por la corriente.

\section{Composición y abundancia de la ictiofauna}

En total se colectaron 4.098 especimenes de peces que fueron asignados a 124 especies, 25 familias y 7 órdenes (ver Tabla 2). Del total de especies, el $56 \%$ pertenecía al orden Characiformes, $22 \%$ a Siluriformes, $15 \%$ a Perciformes, $5 \%$ Gymnotiformes, y $2 \%$ restante a Synbranchiformes, Rajiformes y Beloniformes. Las familias más ricas en especies fueron Characidae con un $36 \%$, seguido por la familia Cichlidae con 15\%, Loricariidae y Curimatidae con $7 \%$ cada una, y Doradidae con 5\%, mientras que 19 familias presentan una baja representación de riqueza de especies por familia, contribuyendo juntas con el 30\% (ver Apéndice).

La diferencia de 'abundancia' o capturabilidad fue notable entre épocas, con 3.481 individuos de 95 especies capturados durante aguas bajas y 617 individuos de 70 especies durante aguas altas. La especie más abundante fue Hemigrammus lunatus, un pequeño carácido o 'sardina' que representó el $18 \%$ de todas las capturas, seguida de Pyrrhulina brevis, Corydoras hastatus Ctenobrycon spilurus y Hemigrammus unilineatus que superaron el 5\% en el total de capturas (Apéndice). La dominancia de estas especies fue notable durante aguas bajas cuando las capturas fueron máximas, pero cambió durante aguas altas ya que Corydoras hastatus no fue capturada, y dos especies no registradas en aguas bajas estuvieron entre las cinco más abundantes (Curimatella alburna y Steindachnerina sp.). Otra diferencia estacional fue la ocurrencia de especies exclusivas de

Tabla 1. Medición de las características físico-químicas del Río Paraguá.

Table 1. Measurement of physical and chemical characteristics of the Paraguá River.

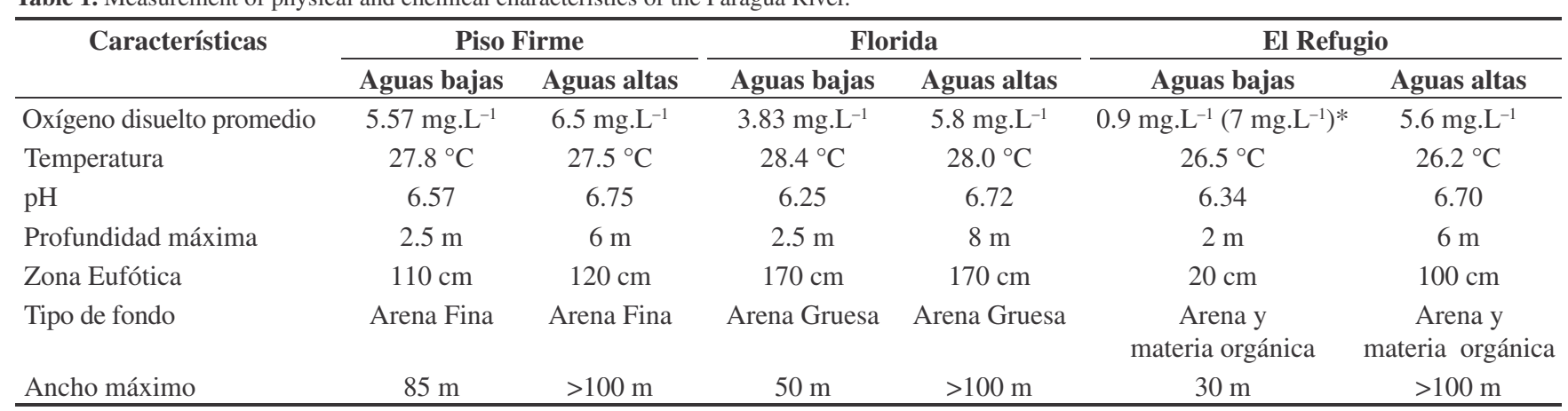


aguas bajas (53 especies) como Apistogramma trifasciata, Astyanax cf. paranahybae Gnatocharax steindachneri, y Gasteropelechus sternicla, y otras exclusivas de aguas altas (23 especies) como Curimatella dorsalis, C. alburna, Eigenmannia melanopogon, Hemigrammus gracilis, Astyanax abramis, y Metynnis luna. Esta diferencia estacional se debió principalmente a los muestreos de Piso Firme y Florida, ya que en El Refugio por su menor riqueza sólo hubo 1-5 especies exclusivas por época.

La riqueza de especies fue disminuyendo río arriba, desde un total de 90 especies, 20 familias y 7 órdenes en Piso Firme (859 ind), a 71 especies, 20 familias y 6 órdenes en Florida (2991 ind), hasta 23 especies, 7 familias y 3 órdenes en El Refugio (248 ind) (ver Tabla 2). Piso Firme mostró 48 especies exclusivas, entre las que se destacan peces mayores como Pseudoplatystoma fasciatum, Cichla monoculus, Serrasalmus rhombeus, Leporinus friderici, Prochilodus cf. nigricans, Potamotrygon sp. En Florida se registraron 26 especies exclusivas entre las que se destacan Steindachnerina sp., Hemigrammus rhodostomus, H. ocellifer, Poptella orbicularis, y Serrapinus calliurus, entre otras. En El Refugio hubo solo 3 taxones (Rhamdia sp., Crenicichla sp. y Trachelyopterus cf coriaceus) no compartidos con Florida o con Piso Firme. Las especies más abundantes por sitio fueron Moenkhausia colletti, con el $12 \%$ de las capturas de Piso Firme, Hemigrammus lunatus con el $22 \%$ en Florida y Gnactocharax steindachneri con el $31 \%$ en El Refugio. El índice de Sorensen indicó una mayor similitud entre Piso Firme y Florida (0.56); mientras que El Refugio tuvo poco en común con Piso Firme (0.15) o con Florida (0.18).

\section{Discusión}

\section{Actualizaciones al conocimiento de la ictiofauna}

Las 123 especies inventariadas aumentan a 315 especies la riqueza conocida para la cuenca del Río Iténez en Bolivia y la convierten en la segunda cuenca más diversa del país después de la del Mamoré (327 especies). Combinando este inventario con las 39 especies registradas por Sarmiento (1998) en El Refugio, la riqueza del Río Paraguá alcanzaría a 152 especies, correspondientes a 28 familias y 9 órdenes.
De este total, 35 son nuevos registros para el Parque Noel Kempff Mercado y diez son nuevos para la cuenca amazónica boliviana (Anostomoides sp, Astyanax cf. paranahybae, Curimatopsis cripticus, Hemigrammus rhodostomus, $H$. gracilis, Mycroschemobrycon cf. casiquiare, Moenkhausia cf. affinis, Microsternachus sp, Acanthodoras cataphractus y Glyptoperichthys gibbiceps). Con estas adiciones, el registro de 635 especies en la ictiofauna boliviana (Sarmiento y Barrera, 2003) se incrementaría a 642. Llama la atención, la ocurrencia de taxones como Astyanax cf. paranahybae, Crenichla cf semifasciata, C. cf. jupiaensis, Aphyocharax paraguayensis, Metynnis luna, M. argenteus, Serrapinus calliurus y Liposarcus anisitsi que fueran antes asignados sólo para la cuenca Del Plata (Gery 1977), lo que permite suponer la existencia de una comunicación reciente con la cuenca Amazónica.

\section{Composición íctica y características ambientales}

Las características físicas, químicas y biológicas del río Paraguá coinciden en gran parte con la condición de 'aguas negras' de la clasificación de Sioli (1975), excepto por sus elevados valores de pH (Tabla 3) Uno de los rasgos de su ictiofauna fue la mayor riqueza de especies de la familia Cichlidae (18 spp) en comparación con la de la familia Pimelodidae (3) que predomina en ríos de aguas blancas. Esto puede obedecer a la adaptación de los cíclidos a aguas de escasa corriente y turbidez, ya que dependen de su vista para su alimentación, mientras que los pimelódidos que habitan preferentemente ríos con sedimentos cuentan con barbillas y órganos anexos para detectar su alimento (Lagler 1984, Nelson 1999).

Los sitios de Piso Firme y Florida, ambos rodeados por bosque, mostraron mayor diversidad de peces y similitud entre sí que con El Refugio, ubicado río arriba y rodeado por sabanas. La riqueza y abundancia en El Refugio fue notoriamente menor que en los otros sitios, y esto podría deberse al menor caudal y heterogeneidad ambiental de su entorno. Las especies mayores pescadas para consumo local y comercio (principalmente Pseudoplatystoma, Cichla, Metynnis, y Serrasalmus; Muñoz 2006) se encuentran en el área de Piso Firme, algunas grandes también llegan hasta Florida (Serrasalmus, Cichla,), pero sólo pocas con cierto valor de consumo (Hoplias, Hoplerytrinus)

Tabla 2. Resumen de la fauna íctica registrada en el Río Paraguá.

Table 2. Summary of the fish fauna recorded in the Paraguá River.

\begin{tabular}{|c|c|c|c|c|c|c|}
\hline & Piso Firme & Florida & El Refugio & Aguas altas & Aguas bajas & Total general \\
\hline $\mathrm{N}^{\circ}$ individuos & 859 & 2991 & 248 & 617 & 3481 & 4098 \\
\hline $\mathrm{N}^{\circ}$ Especies & 90 & 71 & 23 & 70 & 95 & 124 \\
\hline $\mathrm{N}^{\mathrm{o}}$ Ordenes & 7 & 6 & 3 & 6 & 6 & 7 \\
\hline
\end{tabular}

Tabla 3. Comparación de características de los ríos amazónicos (Sioli 1975) vs. el Río Paraguá.

Table 3. Comparison of characteristics of Amazonian rivers (Sioli 1975) vs. the Paraguá River.

\begin{tabular}{|c|c|c|c|c|}
\hline \multirow[t]{2}{*}{ Parámetros } & \multicolumn{3}{|c|}{ Tipos de ríos (Sioli 1975) } & \multirow{2}{*}{$\begin{array}{c}\text { Río Paragua } \\
\text { (en este estudio) }\end{array}$} \\
\hline & Aguas claras & Aguas blancas & Aguas negras & \\
\hline $\mathrm{pH}$ & $4.5-7.8$ & $6.2-7.2$ & $3.8-4.9$ & $6.4-6.7$ \\
\hline Turbidez & Baja & Alta & Baja & Baja \\
\hline Oxígeno disuelto & Alto & Moderado & Bajo & Bajo \\
\hline Nutrientes & Bajos & Altos & Bajo & Bajo \\
\hline Riqueza de Pimelodidae & Baja & Alta & Baja & Baja \\
\hline Riqueza de Cichlidae & Baja & Baja & Alta & Alta \\
\hline
\end{tabular}


hasta El Refugio. En este sitio, tanto en nuestros muestreos como en los de Sarmiento (1998), faltaron las familias Curimatidae y Gasteropelecidae, y especies como Hemigrammus bellottii y H. rhodostomus que sí llegan a Florida. La especie Gnathocharax steindachneri fue la más abundante en el Refugio pero no así en Florida y Piso Firme. De los tres sitios de estudio sobresalen especies de pequeño porte y con potencial para la acuarofilia como Laemolita taeniata y Potamorrhaphis eigenmanni y otras de los géneros Nannostomus, Pyrrhulina, Carnegiella y Gasteropelechus.

Otra razón para la menor riqueza en El Refugio es que la concentración de oxígeno disuelto llega a ser crítica durante aguas bajas. Cuando el río disminuye su caudal y no corre, las macrófitas flotantes pueden cubrir todo el espejo de agua y reducir drásticamente la disponibilidad de oxígeno para los peces porque impiden la acción del viento en la superficie, hacen 'sombra' a la zona eufótica evitando que las algas produzcan oxígeno, y generan detritos que consumen oxígeno en su descomposición. Afortunadamente, algunos meandros anchos pueden quedar aislados sin muchas plantas y sirven de refugio para peces que si no morirían antes de que comiencen las lluvias. En la época seca, la gente local también realiza quemas de sabanas para favorecer el pastoreo, y a menudo las primeras lluvias llevan una considerable carga de cenizas al río. De acuerdo a versiones de los lugareños, estas quemas producen grandes mortandades de peces entre El Refugio y Florida, aunque durante el estudio esto no fue constatado.

La proporción de nuevos registros producidos por este inventario sugiere que el conocimiento de la ictiofauna de la cuenca del Iténez es incipiente, y que es muy probable que arroyos, bahías y otros cuerpos de agua del sistema del Río Paraguá contengan especies no registradas o aún desconocidas. Las causales de la distribución, abundancia y desaparición temporal de estas especies también esta poco entendida, y es importante establecer la relación entre la ganadería, las quemas y la mortalidad estacional de peces en el Río Paraguá. Los planes de manejo pesquero son un inicio para encarar seriamente este problema, y deberían incorporar mecanismos de monitoreo de las poblaciones de peces por los mismos pescadores, combinados con el registro de datos ambientales para entender y poder prevenir impactos negativos sobre estos recursos.

\section{Agradecimientos}

Al American Museum of Natural History por el aporte financiero y asesoría para el trabajo de campo y al Museo NKM, Universidad Autónoma Gabriel René Moreno, y Wildlife Conservation Society por el respaldo científico para completar y publicar este estudio de tesis. El SERNAP y la dirección del Parque Nacional NKM autorizaron y facilitaron las colectas realizadas con la valiosa ayuda de Osvaldo Maillard y Mauricio Herrera del Museo NKM. También queremos agradecer al Departamento de Geografía e Informática del Museo NKM., al proporcionar el mapa del área de trabajo.

\section{Referencias Bibliográficas}

EIGENMANN, C. \& EIGENMANN, R. 1890. South American Nematognathi. California Academy of Sciences, San Francisco, CA. GERY, J. 1977 Characoides of the World. T.F.H. Publ., Neptune City.GOULDING, M. 1980 The Fishes and the Forest. University of California Press; Berkeley.
KULLANDER, S. 1986 Cichlid fishes of the Amazon River drainage of Peru. Stockholm, Sweden.

LAGLER, K., BARDACH, J., MILLER, R. \& MAY PASSINO, D. (eds) 1984. Ictiología. Primera Edición en Español. México, D.C.

LAUZANnE, L. \& LOUBENS, G. 1985. Peces del Mamoré. Orstom. Trinidad.

MACHADO-ALLISON, A. \& FINK, W. 1995. Sinopsis de las especies de la subfamilia Serrasalminae presentes en la cuenca del Orinoco: claves, diagnosis e ilustraciones. Universidad Central de Venezuela. Caracas.

MAGO-LECCIA, F. 1994. Peces eléctricos de las aguas continentales de América: clasificación y catálogo de los peces eléctricos del Orden Gymnotiformes (Teleostei: Ostariophysi), incluyendo la descripción de nuevos géneros y especies. Instituto de Zoología Tropical. Caracas, Venezuela. p. 1-46.

MAGURRAN, A. 1989. Diversidad ecología y medición. Ediciones Vedra. Barcelona.

MALABARBA, L. R., REIS, R. E., VARI, R. P., LUCENA, Z. M. \& LUCENA, C. A. (eds) 1998. Phylogeny and Classification of Neotropical Fishes. Porto Alegre.

MUÑOZ, H. 2006. Biología del tucunaré (Cichla aff. monoculus) y pesca artesanal en el río Bajo Paraguá, Santa Cruz, Bolivia. Revista Boliviana Ecología y Conservación 19:89-99

NAVARRO, G. \& MALDONADO, M. 2002. Biogeografía de Bolivia y ambientes acuáticos de Bolivia. Ed. Fundación Simón I. Patiño. Cochabamba.

NELSON, J.S. 1999. Fishes of the World. John Wiley \& Sons, Inc. New York.

SARMIENTO, J.1998. Ictiología del PNNKM. pp. 174-180. En: KILLEEN, T. J., Y T. S. SCHULENBERG (eds.) A Biological Assessment of Parque Nacional Noel Kempff Mercado, Bolivia. RAP. Working Papers 10, Conservation International, Washington, D.C.

SARMIENTO, J. \& BARRERA, S. 2003. Peces, pp.126-133; 574-582 en Ibisch P.L. y G. Mérida (eds.) Biodiversidad: La riqueza de Bolivia. Estado de conocimiento y conservación. Ministerio de Desarrollo Sostenible. Editorial FAN, Santa Cruz De La Sierra.

SCHAEFER, S. 2000. Fishes of innundated tropical savannas: diversity and endemism in the Serrania Huanchaca of Eastern Bolivia. American Museum of Natural History New York.

SIOLI, H. 1975. River Ecology. Edited by Whitton Ecol. Stud. 2:461-468.

VAN DAMME, P.A. 2001. Plan de manejo de los recursos pesqueros del Río Paraguá (Bajo Paraguá). Unidad de Limnología y Recursos Acuáticos UMSS. Santa Cruz.

VARI, R. 1984. Systematics of the Neotropical characiform genus Potamorhina (Pisces: Ostariophysi), Smithsonian Contributions to Zoology, 400, 1-36.

VARI, R. 1991. Systematics of the Neotropical characiform genus Steindachnerina Fowler (Pisces: Ostariophysi), Smithsonian Contributions to Zoology, 507, 1-118.

VARI, R. 1992. Systematics of the Neotropical characiform genus Curimatella Eigenmann and Eigenmann (Pisces: Ostariophysi), with summary comments on the Curimatidae. Smithsonian Contributions to Zoology, $533,1-48$. 


\section{Apéndice}

Tabla 1. Especies y número de individuos capturados en este estudio por sitio y época.

Table 1. Species and number of individuals captured in this study per site and season.

\begin{tabular}{|c|c|c|c|c|c|c|c|c|}
\hline No. & Especies & Piso Firme & Florida & El Refugio & Aguas altas & Aguas bajas & Total indiv. & $\%$ indiv. \\
\hline & \multicolumn{8}{|l|}{ RAJIFORMES } \\
\hline & \multicolumn{8}{|l|}{ Dasyatidae } \\
\hline \multicolumn{9}{|c|}{ LEPIDOSIRENIFORMES } \\
\hline \multicolumn{9}{|c|}{ Lepidosirenidae } \\
\hline 2 & Lepid osiren paradoxa ${ }^{\mathrm{a}}$ & - & - & $\mathrm{x}$ & - & - & - & - \\
\hline \multicolumn{9}{|c|}{ CHARACIFORMES } \\
\hline \multicolumn{9}{|c|}{ Erythrinidae } \\
\hline 3 & Hoplerytrinus unitaeniatus & 1 & 4 & 2 & 1 & 6 & 7 & 0,17 \\
\hline 4 & Hoplias malabaricus ${ }^{\mathrm{a}}$ & 16 & 18 & 2 & 7 & 29 & 36 & 0,88 \\
\hline \multicolumn{9}{|c|}{ Lebiasinidae } \\
\hline 5 & Nannostomus harrisoni ${ }^{\mathrm{a}}$ & 6 & 0 & 0 & 0 & 6 & 6 & 0,15 \\
\hline 7 & Pyrrhulina australis ${ }^{\mathrm{a}}$ & - & & $\mathrm{x}$ & - & - & - & - \\
\hline 8 & Pyrrhulina brevis & 17 & 403 & 28 & 57 & 391 & 448 & 10,93 \\
\hline \multicolumn{9}{|c|}{ Anostomidae } \\
\hline 9 & Anostomoides sp. ${ }^{\mathrm{b}}$ & 0 & 1 & 0 & 0 & 1 & 1 & 0,02 \\
\hline 10 & Laemolyta taeniata & 3 & 0 & 0 & 0 & 3 & 3 & 0,07 \\
\hline 11 & Leporinus friderici & 3 & 0 & 0 & 3 & 0 & 3 & 0,07 \\
\hline 12 & Leporinus sp. ${ }^{\mathrm{a}}$ & - & - & $\mathrm{x}$ & - & - & - & - \\
\hline 13 & Schizodon fasciatum & 2 & 0 & 0 & 1 & 1 & 2 & 0,05 \\
\hline \multicolumn{9}{|c|}{ Hemiodidae } \\
\hline 14 & Hemiodopsis semitaeniatus & 2 & 0 & 0 & 2 & 0 & 2 & 0,05 \\
\hline \multicolumn{9}{|c|}{ Curimatidae } \\
\hline 15 & Curimatella alburna & 1 & 55 & 0 & 56 & 0 & 56 & 1,37 \\
\hline 18 & Curimatella cf. inmaculata & 0 & 1 & 0 & 1 & 0 & 1 & 0,02 \\
\hline 19 & Curimatopsis crypticus ${ }^{\mathrm{b}}$ & 1 & 0 & 0 & 1 & 0 & 1 & 0,02 \\
\hline 20 & Cyphocharax spilurus & 82 & 1 & 29 & 3 & 109 & 112 & 2,73 \\
\hline 21 & Eigenmannina melanopogon & 6 & 0 & 0 & 6 & 0 & 6 & 0,15 \\
\hline 22 & Steindachnerina sp. & 0 & 50 & 0 & 50 & 0 & 50 & 1,22 \\
\hline \multicolumn{9}{|c|}{ Prochilodontidae } \\
\hline 23 & Prochilodus cf. nigricans & 2 & 0 & 0 & 0 & 2 & 2 & 0,05 \\
\hline \multicolumn{9}{|c|}{ Crenuchidae } \\
\hline 24 & Characidium sp. & 0 & 3 & 0 & 3 & 0 & 3 & 0,07 \\
\hline 25 & Jobertina lateralis ${ }^{\mathrm{a}}$ & & & $\mathrm{x}$ & & & & \\
\hline \multicolumn{9}{|c|}{ Gasteropelecidae } \\
\hline 26 & Carnegiella marthae & 27 & 5 & 0 & 25 & 7 & 32 & 0,78 \\
\hline 27 & Carnegiella strigata & 4 & 8 & 0 & 1 & 11 & 12 & 0,29 \\
\hline 28 & Gasteropelechus sternicla & 4 & 31 & 0 & 0 & 35 & 35 & 0,85 \\
\hline \multicolumn{9}{|c|}{ Cynodontidae } \\
\hline 29 & Hydrolychus scomberoides & 2 & 0 & 0 & 0 & 2 & 2 & 0,05 \\
\hline \multicolumn{9}{|c|}{ Characidae } \\
\hline 30 & Acestrorhynchus microlepis & 2 & 0 & 0 & 2 & 0 & 2 & 0,05 \\
\hline 31 & Acestrorhynchus falcirostris & 0 & 1 & 0 & 0 & 1 & 1 & 0,02 \\
\hline 32 & Aphyocharax paraguayensis ${ }^{\mathrm{c}}$ & 5 & 0 & 0 & 0 & 5 & 5 & 0,12 \\
\hline 33 & Astyanax abramis & 0 & 5 & 0 & 5 & 0 & 5 & 0,12 \\
\hline
\end{tabular}


Tabla 1. Continuación...

\begin{tabular}{|c|c|c|c|c|c|c|c|c|}
\hline No. & Especies & Piso Firme & Florida & El Refugio & Aguas altas & Aguas bajas & Total indiv. & $\%$ indiv. \\
\hline 34 & Astyanax guaporensis & 15 & 0 & 0 & 13 & 2 & 15 & 0,37 \\
\hline 35 & Astyanax paucidens & 39 & 0 & 0 & 0 & 39 & 39 & 0,95 \\
\hline 36 & Astyanax cf. paranahybae & 5 & 101 & 0 & 0 & 106 & 106 & 2,59 \\
\hline 37 & Astyanax sp. & 1 & 0 & 0 & 1 & 0 & 1 & 0,02 \\
\hline 38 & Brycon sp. & 7 & 0 & 0 & 0 & 7 & 7 & 0,17 \\
\hline 39 & Bryconops caudomaculatus & 4 & 1 & 0 & 3 & 2 & 5 & 0,12 \\
\hline 40 & Cataprion mento & 3 & 0 & 0 & 2 & 1 & 3 & 0,07 \\
\hline 41 & Charax gibbosus ${ }^{\mathrm{a}}$ & - & - & $\mathrm{x}$ & - & - & - & - \\
\hline 42 & Cheirodon sp. ${ }^{\mathrm{a}}$ & - & - & $\mathrm{x}$ & - & - & - & - \\
\hline 43 & Cheirodontinae sp. ${ }^{\mathrm{a}}$ & - & - & $\mathrm{x}$ & - & - & - & - \\
\hline 44 & Ctenobrycon spilurus & 20 & 231 & 6 & 28 & 229 & 257 & 6,27 \\
\hline 45 & Deuterodon sp. & 4 & 0 & 0 & 0 & 4 & 4 & 0,10 \\
\hline 46 & Eucynopotamus sp. ${ }^{\mathrm{a}}$ & - & - & $\mathrm{x}$ & - & - & - & - \\
\hline 47 & Gnacthocharax steindachneri & 2 & 1 & 77 & 0 & 80 & 80 & 1,95 \\
\hline 48 & Gymnocorymbus ternetzi & 0 & 1 & 17 & 2 & 16 & 18 & 0,44 \\
\hline 49 & Hemigrammus bellottii & 22 & 10 & 0 & 14 & 18 & 32 & 0,78 \\
\hline 50 & 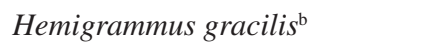 & 6 & 0 & 0 & 6 & 0 & 6 & 0,15 \\
\hline 51 & Hemigrammus lunatus & 78 & 652 & 13 & 54 & 689 & 743 & 18,12 \\
\hline 52 & Hemigrammus rhodostomus ${ }^{\mathrm{b}}$ & 0 & 27 & 0 & 0 & 27 & 27 & 0,66 \\
\hline 53 & Hemigrammus ocellifer & 0 & 17 & 10 & 0 & 27 & 27 & 0,66 \\
\hline 54 & Hemigrammus sp. & 0 & 18 & 15 & 2 & 31 & 33 & 0,80 \\
\hline 55 & Hemigrammus unilineatus ${ }^{\mathrm{a}}$ & 22 & 196 & 5 & 31 & 192 & 223 & 5,44 \\
\hline 56 & Hyphessobrycon callistus ${ }^{\mathrm{a}}$ & 18 & 0 & 3 & 1 & 20 & 21 & 0,51 \\
\hline 57 & Hyphessobrycon sp. ${ }^{\mathrm{a}}$ & - & - & $\mathrm{x}$ & - & - & - & - \\
\hline 58 & Iguanodectes spilurus ${ }^{\mathrm{a}}$ & 51 & 4 & $\mathrm{x}$ & 23 & 32 & 55 & 1,34 \\
\hline 59 & Knodus sp. & 2 & 0 & 0 & 0 & 2 & 2 & 0,05 \\
\hline 60 & Metynnis argenteus ${ }^{\mathrm{b}}$ & 1 & 0 & 0 & 0 & 1 & 1 & 0,02 \\
\hline 61 & Metynnis luna ${ }^{\mathrm{b}}$ & 4 & 0 & 0 & 4 & 0 & 4 & 0,10 \\
\hline 62 & Hyphessobrycon megalopterus & 0 & 13 & 0 & 1 & 12 & 13 & 0,32 \\
\hline 63 & Megalamphodus sp. ${ }^{\mathrm{a}}$ & - & - & $\mathrm{x}$ & - & - & - & - \\
\hline 64 & Microschemobrycon casiquiare ${ }^{\mathrm{b}}$ & 1 & 0 & 0 & 1 & 0 & 1 & 0,02 \\
\hline 65 & Moenkhausia cf. affinis ${ }^{\mathrm{b}}$ & 0 & 1 & 0 & 1 & 0 & 1 & 0,02 \\
\hline 66 & Moenkhausia colletti & 107 & 8 & 0 & 27 & 88 & 115 & 2,80 \\
\hline 67 & Moenkhausia cotinho & 14 & 0 & 0 & 0 & 14 & 14 & 0,34 \\
\hline 68 & Moenkhausia dichroura $^{\mathrm{a}}$ & - & - & $\mathrm{x}$ & - & - & - & - \\
\hline 69 & Moenkhausia intermedia & 18 & 102 & 0 & 22 & 98 & 120 & 2,93 \\
\hline 70 & Moenkhausia lepidura & 20 & 1 & 0 & 16 & 5 & 21 & 0,51 \\
\hline 71 & Moenkhausia sanctaefilomenae & 14 & 154 & 4 & 12 & 160 & 172 & 4,20 \\
\hline 72 & Serrapinnus calliurus ${ }^{\mathrm{c}}$ & 0 & 6 & 0 & 0 & 6 & 6 & 0,20 \\
\hline 73 & Phenacogaster cf. pectinatus ${ }^{\mathrm{c}}$ & 1 & 0 & 0 & 0 & 1 & 1 & 0,02 \\
\hline 74 & Poptella orbicularis & 0 & 23 & 0 & 8 & 15 & 23 & 0,56 \\
\hline 75 & Pygocentrus nattereri & 1 & 0 & 0 & 0 & 1 & 1 & 0,02 \\
\hline 76 & Roestes cf. molossus & 1 & 0 & 0 & 0 & 1 & 1 & 0,02 \\
\hline 77 & Serrasalmus rhombeus & 7 & 0 & 0 & 2 & 5 & 7 & 0,17 \\
\hline 78 & Serrasalmus spilopleura & 12 & 5 & 0 & 6 & 11 & 17 & 0,41 \\
\hline 79 & Serrasalmus sp. ${ }^{\mathrm{a}}$ & - & - & $\mathrm{x}$ & - & - & - & - \\
\hline 80 & Thayeria obliqua ${ }^{\mathrm{c}}$ & 12 & 0 & 0 & 0 & 12 & 12 & 0,29 \\
\hline 81 & Tetragonopterus chlaceus & 3 & 0 & 0 & 3 & 0 & 3 & 0,07 \\
\hline 82 & Triportheus angulatus ${ }^{\mathrm{a}}$ & - & - & $\mathrm{x}$ & - & - & - & - \\
\hline \multicolumn{9}{|c|}{ GYMNOTIFORMES } \\
\hline \multicolumn{9}{|c|}{ Gymnotidae } \\
\hline 83 & 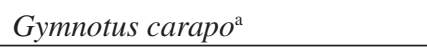 & - & - & $\mathrm{x}$ & - & - & - & - \\
\hline
\end{tabular}


Tabla 1. Continuación...

\begin{tabular}{|c|c|c|c|c|c|c|c|c|}
\hline No. & Especies & Piso Firme & Florida & El Refugio & Aguas altas & Aguas bajas & Total indiv. & $\%$ indiv. \\
\hline \multicolumn{9}{|c|}{ Hypopomidae } \\
\hline 84 & Brachyhypopomus brevirostris ${ }^{\mathrm{c}}$ & 5 & 1 & 0 & 3 & 3 & 6 & 0,15 \\
\hline 85 & Hypopygus lepturus $\mathrm{s}^{\mathrm{a}}$ & 1 & 0 & $\mathrm{x}$ & 1 & 0 & 1 & 0,02 \\
\hline 86 & Hypopomus artedic & 0 & 2 & 0 & 0 & 2 & 2 & 0,05 \\
\hline 87 & Hypopomus sp. $1^{\mathrm{a}}$ & - & - & $\mathrm{x}$ & - & - & - & - \\
\hline 88 & Hypopomus sp. $2^{\mathrm{a}}$ & - & - & $\mathrm{x}$ & - & - & - & - \\
\hline 89 & Microsternachus sp. ${ }^{\mathrm{b}}$ & 2 & 4 & 0 & 0 & 6 & 6 & 0,15 \\
\hline \multicolumn{9}{|c|}{ Sternopygidae } \\
\hline 90 & Eigenmannia trilineata ${ }^{\mathrm{c}}$ & 19 & 27 & 0 & 8 & 38 & 46 & 1,12 \\
\hline 91 & Eigenmannia cf. virescens ${ }^{\mathrm{a}}$ & - & - & $\mathrm{x}$ & - & - & - & - \\
\hline 92 & Sternopygus macrurus ${ }^{\mathrm{a}}$ & - & - & $\mathrm{x}$ & - & - & - & - \\
\hline \multicolumn{9}{|c|}{ Rhamphichthyidae } \\
\hline 93 & Ramphichthys rostratus ${ }^{\mathrm{a}}$ & - & - & $\mathrm{x}$ & - & - & - & - \\
\hline \multicolumn{9}{|c|}{ Electrophoridae } \\
\hline 94 & Electrophorus electricus ${ }^{\mathrm{c}}$ & 1 & 1 & 0 & 1 & 1 & 2 & 0,05 \\
\hline \multicolumn{9}{|c|}{ SILURIFORMES } \\
\hline \multicolumn{9}{|c|}{ Aspredinidae } \\
\hline 95 & Dysichthys sp. & 0 & 1 & 0 & 0 & 1 & 1 & 0,02 \\
\hline \multicolumn{9}{|c|}{ Auchenipteridae } \\
\hline 96 & Ageneiosus inermis ${ }^{\mathrm{c}}$ & 0 & 1 & 0 & 0 & 1 & 1 & 0,02 \\
\hline 97 & Auchenipterichthys thoracatus & 2 & 0 & 0 & 2 & 0 & 2 & 0,05 \\
\hline 98 & Tatia aulopygia & - & - & $\mathrm{x}$ & - & - & - & - \\
\hline 99 & Trachelyopterus cf. coriaceus ${ }^{\mathrm{c}}$ & 0 & 0 & 7 & 0 & 7 & 7 & 0,17 \\
\hline \multicolumn{9}{|c|}{ Callichthyidae } \\
\hline 100 & Corydoras aeneus & 0 & 2 & 0 & 0 & 2 & 2 & 0,05 \\
\hline 101 & Corydoras hastatus ${ }^{\mathrm{a}}$ & 1 & 265 & $\mathrm{x}$ & 0 & 266 & 266 & 6,49 \\
\hline 102 & Hoplosternum littorale ${ }^{\mathrm{a}}$ & 1 & 30 & $\mathrm{x}$ & 0 & 31 & 31 & 0,76 \\
\hline 103 & Megalechis thoracata & 1 & 1 & 0 & 2 & 0 & 2 & 0,05 \\
\hline \multicolumn{9}{|c|}{ Doradidae } \\
\hline 104 & Acanthodoras cataphractus ${ }^{\mathrm{b}}$ & 1 & 0 & 0 & 0 & 1 & 1 & 0,02 \\
\hline 105 & Acanthodoras spinosissimus ${ }^{\mathrm{a}}$ & - & - & $\mathrm{x}$ & - & - & - & - \\
\hline 106 & Amblyodoras hancocki $\mathrm{i}^{\mathrm{a}}$ & - & - & $\mathrm{x}$ & - & - & - & - \\
\hline 107 & Anadoras weddellii ${ }^{\mathrm{c}}$ & 0 & 1 & 0 & 0 & 1 & 1 & 0,02 \\
\hline 108 & Anadoras sp. & 0 & 1 & 0 & 0 & 1 & 1 & 0,02 \\
\hline 109 & Doras eigenmannic & 0 & 1 & 0 & 0 & 1 & 1 & 0,02 \\
\hline 110 & Doras fimbriatus ${ }^{\mathrm{c}}$ & 1 & 0 & 0 & 1 & 0 & 1 & 0,02 \\
\hline 111 & Platydoras costatus & 0 & 3 & 0 & 0 & 3 & 3 & 0,07 \\
\hline \multicolumn{9}{|c|}{ Loricariidae } \\
\hline 112 & Glyptoperichthys lituratus & 0 & 1 & 0 & 0 & 1 & 1 & 0,02 \\
\hline 113 & Glyptoperichthys gibbiceps ${ }^{\mathrm{b}}$ & 1 & 0 & 0 & 0 & 1 & 1 & 0,02 \\
\hline 114 & Glyptoperichthys sp. & 0 & 1 & 0 & 0 & 1 & 1 & 0,02 \\
\hline 115 & Hemiodontichthys acipenserinus & 2 & 0 & 0 & 2 & 0 & 2 & 0,05 \\
\hline 116 & Hypostomus sp. & 1 & 0 & 0 & 1 & 0 & 1 & 0,02 \\
\hline 117 & Hypoptopoma gulare & 2 & 0 & 0 & 0 & 2 & 2 & 0,05 \\
\hline 118 & Liposarcus anisitsi $^{\mathrm{c}}$ & 0 & 1 & 0 & 0 & 1 & 1 & 0,02 \\
\hline 119 & Rineloricaria parva ${ }^{\mathrm{c}}$ & 1 & 9 & 0 & 1 & 9 & 10 & 0,24 \\
\hline 120 & Rineloricaria lima ${ }^{\mathrm{c}}$ & 6 & 0 & 0 & 1 & 5 & 6 & 0,15 \\
\hline 121 & Pterygoplichthys sp. ${ }^{\mathrm{a}}$ & - & - & $\mathrm{x}$ & - & - & - & - \\
\hline \multicolumn{9}{|c|}{ Heptapteridae } \\
\hline 122 & 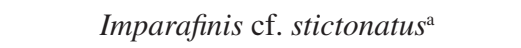 & - & - & $\mathrm{x}$ & - & - & - & - \\
\hline 123 & Rhamdia sp. ${ }^{\mathrm{c}}$ & 0 & 2 & 0 & 0 & 2 & 2 & 0,05 \\
\hline
\end{tabular}


Tabla 1. Continuación...

\begin{tabular}{|c|c|c|c|c|c|c|c|c|}
\hline No. & Especies & Piso Firme & Florida & El Refugio & Aguas altas & Aguas bajas & Total indiv. & $\%$ indiv. \\
\hline \multicolumn{9}{|c|}{ Pimelodidae } \\
\hline 124 & Pimelodus sp. & 0 & 0 & 1 & 0 & 1 & 1 & 0,02 \\
\hline 125 & Pimelodus altipinnis & 1 & 0 & 0 & 1 & 0 & 1 & 0,02 \\
\hline 126 & Pseudoplatystoma fasciatum & 2 & 0 & 0 & 1 & 1 & 2 & 0,05 \\
\hline \multicolumn{9}{|c|}{ Tricomycteridae } \\
\hline 127 & Trichomycterus sp. & 0 & 1 & 0 & 0 & 1 & 1 & 0,02 \\
\hline 128 & Tricomycteridae $^{\mathrm{a}}$ & - & - & $\mathrm{x}$ & - & - & - & - \\
\hline \multicolumn{9}{|c|}{ PERCIFORMES } \\
\hline \multicolumn{9}{|c|}{ Cichlidae } \\
\hline 129 & Acaronia nassa & 0 & 1 & 0 & 0 & 1 & 1 & 0,02 \\
\hline 130 & Aequidens sp. & 15 & 3 & 0 & 16 & 2 & 18 & 0,44 \\
\hline 131 & Aequidens tetramerus & 0 & 4 & 6 & 1 & 9 & 10 & 0,24 \\
\hline 132 & Apistogramma inconspicua & 8 & 28 & 3 & 6 & 33 & 39 & 0,95 \\
\hline 133 & Apistogramma trifasciata ${ }^{\mathrm{c}}$ & 12 & 145 & 2 & 0 & 159 & 159 & 3,88 \\
\hline 134 & Apistogramma sp. $1^{\mathrm{a}}$ & - & - & $\mathrm{x}$ & - & - & - & - \\
\hline 135 & Apistogramma sp. $2^{\mathrm{a}}$ & - & - & $\mathrm{x}$ & - & - & - & - \\
\hline 136 & Astronotus ocellatus $^{\mathrm{c}}$ & 1 & 0 & 0 & 0 & 1 & 1 & 0,02 \\
\hline 137 & Bujurquina sp. ${ }^{c}$ & 2 & 0 & 0 & 0 & 2 & 2 & 0,05 \\
\hline 138 & Cichla monoculus & 10 & 0 & 0 & 0 & 10 & 10 & 0,24 \\
\hline 139 & Cichlasoma amazonarum ${ }^{c}$ & 3 & 4 & 0 & 4 & 3 & 7 & 0,17 \\
\hline 140 & Cichlasoma boliviensis & 5 & 20 & 3 & 3 & 25 & 28 & 0,68 \\
\hline 141 & Crenicara punctulatum $^{c}$ & 1 & 0 & 0 & 1 & 0 & 1 & 0,02 \\
\hline 142 & Crenicichla cf. jupiaensis & 0 & 2 & 0 & 2 & 0 & 2 & 0,05 \\
\hline 143 & Crenicichla semifasciata $^{\mathrm{C}}$ & 5 & 146 & 5 & 8 & 148 & 156 & 3,80 \\
\hline 144 & Crenicichla sp. & 0 & 0 & 1 & 1 & 0 & 1 & 0,02 \\
\hline 145 & Heros sp. ${ }^{\mathrm{a}}$ & 3 & 0 & $\mathrm{x}$ & 0 & 3 & 3 & 0,07 \\
\hline 146 & Laetacara dorsigera & 5 & 79 & 4 & 14 & 74 & 88 & 2,15 \\
\hline 147 & Mesonauta festivus ${ }^{\mathrm{a}}$ & 0 & 0 & $\mathrm{x}$ & 0 & 0 & 0 & 0 \\
\hline 148 & Mesonauta insignis & 5 & 30 & 5 & 9 & 31 & 40 & 0,98 \\
\hline 149 & Satanoperca papaterra & 1 & 0 & 0 & 0 & 1 & 1 & 0,02 \\
\hline \multicolumn{9}{|c|}{ BELONIFORMES } \\
\hline \multicolumn{9}{|c|}{ Belonidae } \\
\hline 150 & Potamorrhaphis eigenmmanni & 9 & 1 & 0 & 5 & 5 & 10 & 0,24 \\
\hline \multicolumn{9}{|c|}{ CYPRINODONTIFORMES } \\
\hline \multicolumn{9}{|c|}{ Rivulidae } \\
\hline 151 & Cynolebias sp. ${ }^{\mathrm{a}}$ & - & - & $\mathrm{x}$ & - & - & - & - \\
\hline \multicolumn{9}{|c|}{ SYNBRANCHIFORMES } \\
\hline \multicolumn{9}{|c|}{ Synbranchidae } \\
\hline 152 & Synbranchus marmoratus ${ }^{\mathrm{a}}$ & 3 & 4 & $\mathrm{x}$ & 2 & 5 & 7 & 0,17 \\
\hline \multicolumn{2}{|r|}{ Total } & 859 & 2991 & 248 & 617 & 3481 & 4098 & 100 \\
\hline
\end{tabular}

a) Registros previos de Sarmiento (1998); b) Nuevos registros para el Parque Noel Kempff; c) Nuevos registros para la Amazonia boliviana.

a) Previous records from Sarmiento (1998); b) New records for Noel Kempff Park; c) New records for the Bolivian Amazon. 